\title{
Sucesión temprana de la taxocenosis Mollusca-Annelida-Crustacea en raíces sumergidas de mangle rojo en San Andrés Isla, Caribe colombiano
}

\author{
Early succession of the taxocenosis Mollusca-Annelida-Crustacea on red mangrove \\ submerged roots in San Andres Island, Colombian Caribbean \\ Patricia E. Romero-Murillo ${ }^{1}$ y Jaime Polanía ${ }^{2}$ \\ ${ }^{1}$ Red de Desarrollo Sostenible. Diagonal 40A No 14-66. Bogotá, Colombia \\ ${ }^{2}$ Instituto de Estudios Ambientales-IDEA Sede Medellín, Calle 59A No 63-20, Medellín, Colombia \\ patolinro@gmail.com
}

\begin{abstract}
The early succession of the taxa Annelida, Mollusca and Crustacea on Rhizophora mangle (red mangrove) submerged roots was analyzed using old branches as ground (which were the experimental units) in San Andres Island. The analysis was related to some physico-chemical and environmental variables during 188 days, between November/ 2002 and May/2003, in Hooker and Honda bays. A total of 34,175 individuals in 130 experimental units were counted, where the annelids represented the largest proportion (77.1\%) from the whole sample. A dendrogram and a nonmetric multidimensional scaling (NMDS) were constructed for each one of the three studied taxa. The analyses showed a weak similarity between the internal part of the Hooker Bay and the remaining stations. Shannon-Wiener diversity, Pielou evenness, Margalef richness and Simpson predominance indexes were calculated throughout the study; the three first indices showed greater values in Hooker Bay than in Honda Bay, while the predominance index was greater in Bay Honda. There were pioneers organisms such as Spirorbis sp., and transient ones, such as crustaceans, whilst molluscs seemed to need longer periods to settle in. These responses reveal that geomorphological characteristics, presence or absence of freshwater currents, tides and rainfall mainly, as well as anthropic factors can influence the development of these communities. Larval dispersion and the proximity to other ecosystems increase or reduce the populations, and only some organisms, adapted to extreme conditions, settle down and maintain in the area. Under favorable conditions, abundance and species diversity tend to increase.
\end{abstract}

Key words: Mangrove roots, Caribbean
Resumen.- Se analizó la sucesión temprana de los taxa Annelida, Mollusca y Crustacea asociados a las raíces sumergidas de Rhizophora mangle (mangle rojo), empleando ramas del mismo como sustrato artificial (unidades experimentales). El análisis se relacionó con algunas variables físico-químicas y ambientales durante 188 días en San Andrés Isla, entre noviembre 2002 y mayo 2003, en tres estaciones de dos bahías, Hooker y Honda. Se identificaron 34.175 individuos en 130 unidades experimentales, los anélidos mostraron la mayor proporción (77,1\%). Se generó un análisis de ordenación y clasificación (dendrograma y un escalamiento multidimensional no métrico-NMDS) para cada uno de los tres taxa estudiados. Los análisis revelaron débil similaridad entre la parte interna de Bahía Hooker y las estaciones restantes. Se calcularon los índices de diversidad de Shannon-Wiener, uniformidad de Pielou, riqueza de Margalef y predominio de Simpson a lo largo del estudio; los tres primeros presentaron mayores valores en Bahía Hooker que en Honda, mientras que el índice de predominio fue mayor en Bahía Honda. Hubo organismos pioneros como el anélido Spirorbis sp. y transitorios como los crustáceos; los moluscos parecieron necesitar periodos más prolongados para asentarse. Estas respuestas revelan que características geomorfológicas, presencia o ausencia de corrientes de agua dulce, mareas y lluvias, principalmente, y factores antrópicos pueden influir en el desarrollo de estas comunidades. Dispersión larval y proximidad con otros ecosistemas aumentan o disminuyen las poblaciones; sólo algunos organismos adaptados a condiciones extremas se establecen y mantienen en el área. Bajo condiciones favorables, la abundancia y diversidad de especies tienden a aumentar.

Palabras clave: Raíces de mangle, Caribe

\section{Introducción}

Los manglares son ecosistemas de litorales, desembocaduras de ríos, lagunas, esteros, terrenos con relieve plano fangoso tropicales y subtropicales (Lacerda et al. 2001). En Colombia cubren $>300.000$ ha distribuidas entre las costas del Mar Caribe (20-30\%) y del Océano Pacífico (70-80\%). La principal especie arbórea es el mangle rojo, Rhizophora mangle (L.), distinguida por sus raíces en zancos, la cual sirve de 
sustrato y hábitat a organismos permanentes o transitorios de moluscos, anélidos y crustáceos, entre otros. Estos grupos pueden ser descritos por sus mecanismos de adaptación a las variaciones de salinidad y temperatura, además de resistir a desecación e inmersión. Así mismo, presentan hábitos incrustantes, perforadores y vágiles (Reyes \& Campos 1992a), y pueden mostrar gradientes en la zonación sobre las raíces (Batista 1980, Farnsworth \& Ellison 1996). Los organismos con hábitos sésiles pueden ser filtradores, herbívoros o depredadores (Farnsworth \& Ellison 1996, Pérez \& Victoria 1980¹) y, a su vez, servir como fuente de alimento para taxa de mayor tamaño (Reyes \& Campos 1992b).

Algunos resultados de los estudios realizados en el archipiélago colombiano de San Andrés, Providencia y Santa Catalina fueron sintetizados en los trabajos de Londoño-Mesa et al. (2002) y Vilardy \& Polanía (2002); Moreno (2002) determinó la composición y la estructura de la comunidad de invertebrados asociados a las raíces del mangle rojo en el complejo de bahías Honda/Hooker, mientras que Romero-Murillo (2004) estudió esta composición en un período de sucesión.

Los organismos asociados a las raíces pueden reflejar los procesos sufridos por el ecosistema, de manera que resulta importante conocerlos y verificar su comportamiento frente a diferentes sucesos naturales o alteraciones ambientales. El presente estudio permite analizar la sucesión temprana durante seis meses, de tres taxa de invertebrados asociada a las raíces sumergidas de $R$. mangle a partir de un sustrato artificial, con relación a la temperatura y la precipitación en San Andrés Isla.

San Andrés Isla es la mayor del Archipiélago de San Andrés, Providencia y Santa Catalina, localizada entre los ( $12^{\circ} 32^{\prime} \mathrm{N}$ y $12^{\circ} 36^{\prime} \mathrm{N}$ y los $81^{\circ} 40^{\prime} \mathrm{O}$ y $\left.81^{\circ} 44^{\prime} \mathrm{O}\right)$ (Fig. 1), a $800 \mathrm{~km}$ de la costa continental colombiana. Su clima es seco tropical y su temperatura media anual $27,4^{\circ} \mathrm{C} \pm 1^{\circ} \mathrm{C}$ (García-Barriga et al. 1985, Garay et al. 1988, Díaz et al. 1996). La temperatura promedio del agua superficial es similar en aguas oceánicas y litorales $\left(28,1 \pm 2^{\circ} \mathrm{C}\right)$ (Díaz et al. 1995). Predominan los vientos alisios del noreste y este-noreste (enero y abril) (GarcíaBarriga et al. 1985, Garay et al. 1988, Díaz et al. 1996). La vegetación es de bosque seco tropical con transición

${ }^{1}$ Pérez ME \& C Victoria. 1980. Aspectos de la comunidad de invertebrados de las raíces sumergidas del mangle rojo en la Bahía de Cartagena. Instituto de Investigaciones Marinas de Punta de Betín (INVEMAR). Seminario Latinoamericano sobre el estudio científico y el impacto humano en el ecosistema de manglares. UNESCO. p. 20. Colombia.

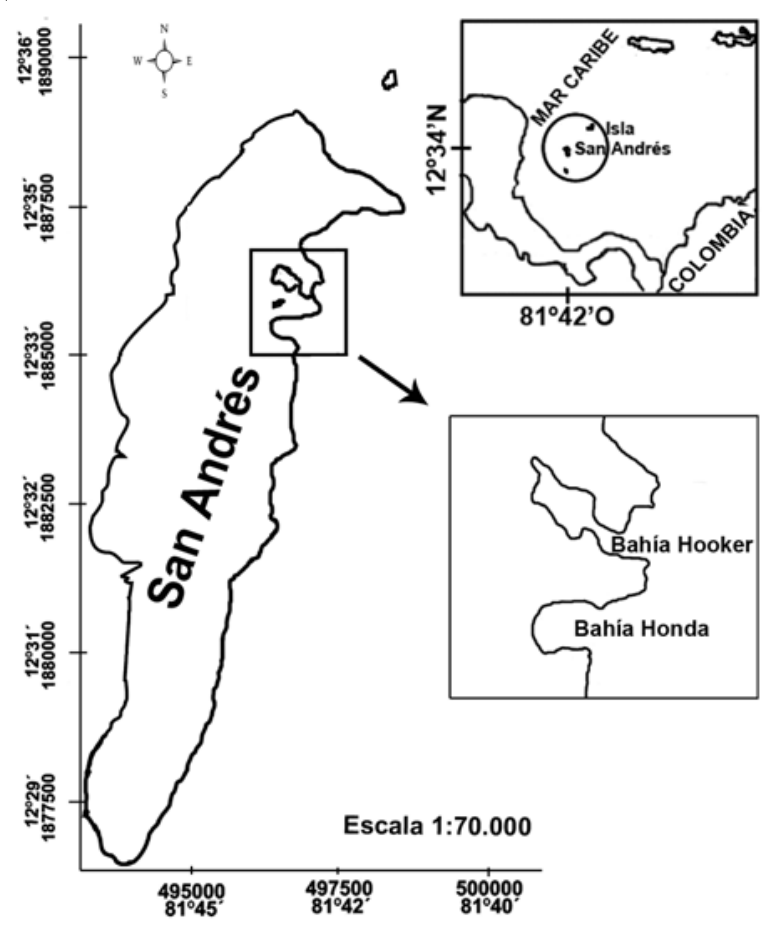

Figura 1

Ubicación del área de estudio en San Andrés Isla (tomado y modificado de CORALINA, 2007)

Study area in San Andrés Island (taken and modified from CORALINA, 2007)

a húmedo (Díaz et al. 1995), con manglares especialmente a barlovento (Buitrago \& Cuartas 2003). No existen corrientes de agua dulce permanentes, deposición de aguas subterráneas por lluvias, pantanos y charcas (García-Barriga et al. 1985).

\section{Material y métodos}

El estudio se efectuó desde el final de la época lluviosa, en noviembre de 2002, hasta mayo de 2003, en dos bahías de la Isla San Andrés (Hooker y Honda). Se evaluó un total de 130 unidades experimentales, ramas de aproximadamente $80 \mathrm{~cm}$ de longitud, recolectadas de árboles vecinos y dispuestas como falsas raíces, amarradas perpendicularmente a las verdaderas sumergidas de cada estación y escogidas al azar, distribuidas en cada una de tres estaciones (interna (1), media (2) y externa (3)) en las bahías Hooker (K) y Honda (O). A los 30 días de montadas las falsas raíces se efectuó un premuestreo, recolectando tres unidades por estación en cada bahía para revisar el inicio del proceso de sucesión, y cuatro unidades para los siguientes muestreos a los 57, 103, 132, 161 y 188 días. En el día 188 sólo se 
tuvieron en cuenta la parte interna y media solamente por agotamiento de montajes en la externa. Las raíces retiradas se colocaron en bolsas plásticas y fueron refrigeradas hasta su procesamiento en laboratorio. En cada muestreo se midieron las variables temperatura del agua y salinidad, y se tuvo en cuenta la precipitación en cada período muestreado.

Los organismos de las raíces fueron separados por grupos (moluscos, crustáceos, y anélidos), fijados en formol al 4\% y luego preservados en alcohol al 70\%. Los grupos se identificaron mediante claves taxonómicas y registros fotográficos hasta el nivel más cercano a especie o, en su defecto, como morfotipo. Se utilizaron claves y referencias para Mollusca (Díaz \& Puyana 1994, González 1998, Victoria \& Pérez 1979), para Crustacea (Bousfield 1973, Victoria \& Pérez 1979, Rodríguez 1980, Ortiz 1994, Gutu \& Sieg 1999, Brusca et al. 2001², Larsen 2002 ${ }^{3}$ ), y para Annelida (Nonato \& Luna 1970, Harper 1971, Fauchald 1975, Fauchald 1977a, b, Victoria \& Pérez 1979, Salazar-Vallejo et al. 1988, Salazar-Vallejo \& Carrera-Parra 1997a, b, De León-González 2001, Rouse \& Pleijel 2001).

Para el tratamiento de los datos se utilizó un análisis multivariado con el programa PRIMER-Plymouth Routines in Multivariate Ecological Research V5 para cada uno de los grupos por muestreo, bahía y estación (Ramírez 1999). Un análisis de clasificación y ordenación utilizó el coeficiente de similaridad de Bray-Curtis; los métodos gráficos del análisis de dendrogramas y de escalamiento multidimensional no métrico (NMDS) agruparon las tres taxa con un porcentaje de similaridad para comparación del $60 \%$ en el dendrograma, y se efectuó un tratamiento individual para cada grupo en el análisis del escalamiento multidimensional no métrico con los siguientes rangos de estrés: menor a 0,01 , gráfica con perfecta representación; entre 0,01 y 0,05, excelente representación; entre 0,05 y 0,1, buena representación con poca probabilidad de mala interpretación; entre 0,1 y 0,2, gráfica de dos dimensiones potencialmente útil. Además, se calcularon los índices ecológicos: riqueza

${ }^{2}$ Brusca RC, V Coelho \& S Taiti. 2001. A guide to the coastal isopods of California. A revision of the family Cirolanidae (Crustacea: Isopoda) with Monographs on Selected Genera http://tolweb.org/treeeukaryotes/animals/arthropoda/crustaea/ isopoda/accesory/caguide/caguide.html $>$ Consultado en junio 2003.

${ }^{3}$ Larsen, K. 2002. Tanaidacea Families. Crustacea. Net. <http:/ /www.crustacea.net/crustace/tanaidacea/>.USA. Consultado el 5 de junio 2003. de Margalef $(\mathrm{d}=(\mathrm{S}-1) / \log (\mathrm{N}))$, uniformidad de Pielou $\left(\mathrm{J}^{\prime}=\mathrm{H}^{\prime} / \mathrm{Log}(\mathrm{S})\right)$, diversidad de Shannon-Wiener con base e $\left(\mathrm{H}^{\prime}=\Sigma\left(\mathrm{Pi}^{*} \log (\mathrm{Pi})\right)\right)$ y predominio de Simpson $\left(\lambda=\Sigma\left(\mathrm{Pi}^{2}\right)\right)$ (Odum 1972), para cada una de las dos bahías, cada una de las tres estaciones en las bahías Hooker y Honda y el período muestreado.

\section{Resultados}

El material recolectado en el premuestreo, realizado el día 30 del experimento incluyó cianobacterias, algas verdes y huevos no identificados, permitió verificar el inicio de la colonización. En el marco de este estudio se recolectaron en total 34.175 individuos, así: 4.676 (13,7\%) pertenecientes a 20 familias de Mollusca; 26.346 (77,1\%) en 13 familias de Annelida; 3.153 (9,2\%) agrupados en 19 familias de Crustacea (Tabla 1). Los taxa más abundantes fueron Spirorbis sp. (24.618 individuos), Petaloconchus sp. (4.059 individuos), Pseudozeuxidae morfo 1 (1.166 individuos).

Las estaciones $\mathrm{O} 1$ y $\mathrm{O} 2$ presentaron fondos de lodos y abundantes conchas vacías, mientras que O3 mostró un sustrato grueso de arenas. En todas las raíces (v.gr. estación K3) mostraron abundantes algas, que no fueron identificadas para este estudio. La estación K2 tenía más de 1,20 m de profundidad y K1 no más de $80 \mathrm{~cm}$. Al caminar sobre el fondo en ambas estaciones se liberaron aceites del sustrato, presumiblemente atrapados allí desde hace más de 20 años, cuando funcionaba cerca la planta eléctrica de la isla.

La precipitación disminuyó de noviembre a mayo (Fig. 2). La salinidad aumentó desde el día 30

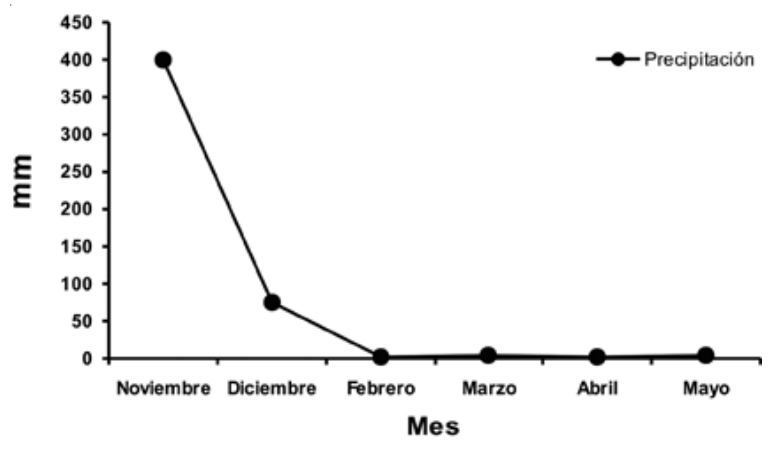

Figura 2

Precipitación de los meses estudiados. Datos suministrados por la Estación Metereológica Aeropuerto Gustavo Rojas Pinilla-IDEAM-San Andrés Isla, 2003

Rainfall during the months of study. Data provided by Meteorological Station Gustavo Rojas Pinilla airportIDEAM-San Andrés Island, 2003 
Vol. 43, No 1,2008

Tabla 1

Mollusca, Annelida y Crustacea recolectados entre febrero y mayo de 2003 en San Andrés Isla, bahías Hooker y Honda

Mollusca, Annelida and Crustacea collected between February and May 2003 on San Andres Island, Hooker and Honda bays

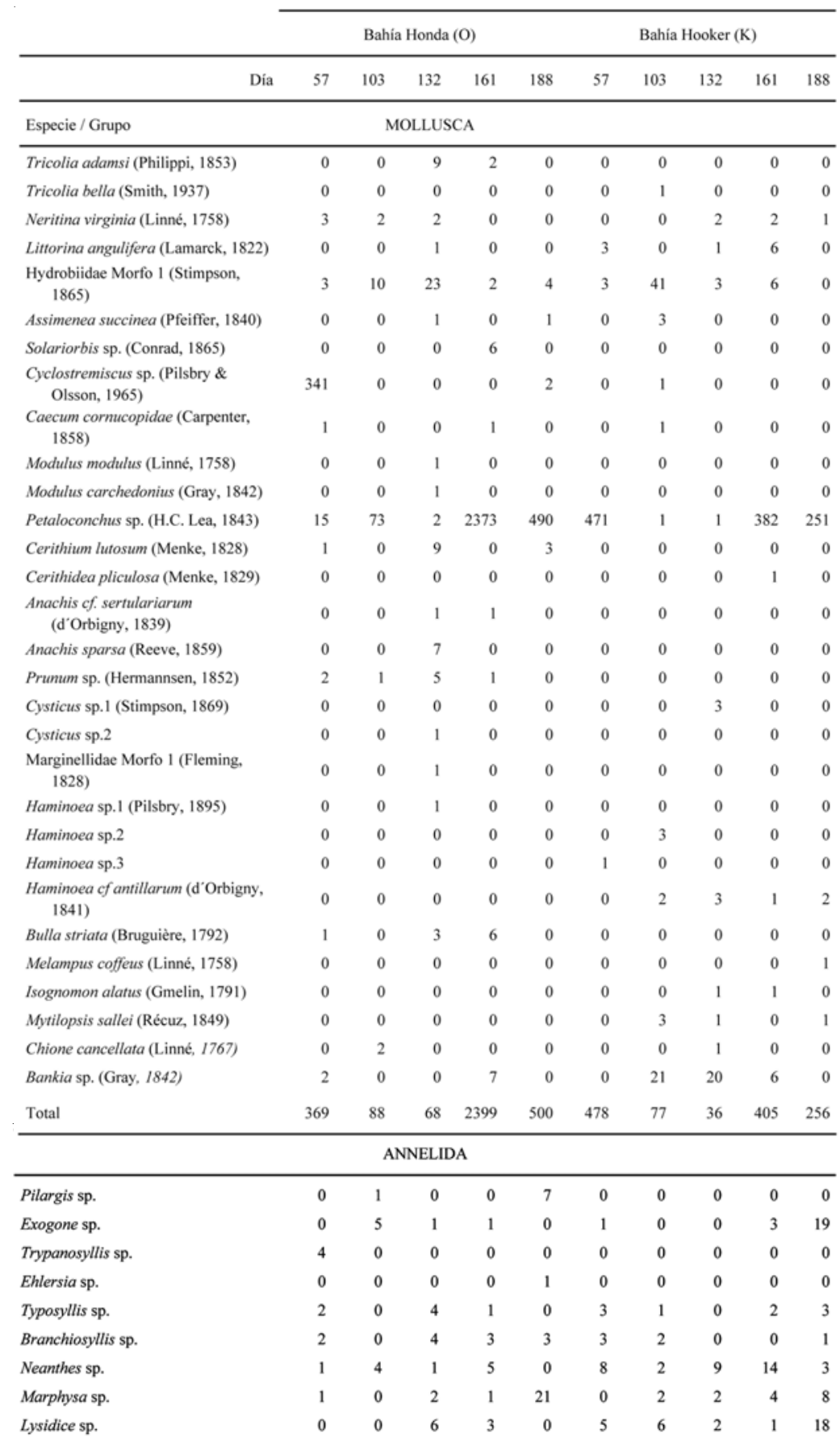


(Cont. Tabla 1)

\begin{tabular}{|c|c|c|c|c|c|c|c|c|c|c|}
\hline Cirratulus spp. & 0 & 0 & 1 & 0 & 2 & 0 & 0 & 0 & 1 & 1 \\
\hline Mediomastus sp. & 0 & 0 & 0 & 0 & 0 & 0 & 0 & 0 & 2 & 0 \\
\hline Heteromastus spp. & 0 & 0 & 0 & 0 & 0 & 2 & 0 & 0 & 1 & 0 \\
\hline Capitella spp. & 0 & 0 & 2 & 1 & 1 & 0 & 0 & 0 & 1 & 0 \\
\hline Anotomastus spp. & 0 & 0 & 0 & 1 & 6 & 0 & 0 & 0 & 0 & 0 \\
\hline Maldanidae Morfo 1 & 0 & 2 & 0 & 0 & 0 & 0 & 0 & 0 & 0 & 0 \\
\hline Armandia sp. & 0 & 1 & 0 & 0 & 0 & 0 & 0 & 0 & 0 & 0 \\
\hline Terebella sp. & 0 & 0 & 0 & 0 & 0 & 0 & 0 & 1 & 0 & 0 \\
\hline Streblosoma sp. & 0 & 0 & 0 & 1 & 0 & 0 & 0 & 0 & 1 & 0 \\
\hline Terebellides sp. & 0 & 1 & 0 & 0 & 0 & 0 & 0 & 0 & 0 & 0 \\
\hline Sabellastarte spp. & 0 & 3 & 7 & 6 & 8 & 54 & 22 & 15 & 97 & 581 \\
\hline Hydroides spp. & 1 & 7 & 22 & 0 & 0 & 5 & 0 & 1 & 3 & 2 \\
\hline Serpulidae Morfo 1. & 54 & 0 & 2 & 39 & 0 & 96 & 18 & 16 & 428 & 6 \\
\hline Spirorbis sp. & 2224 & 2060 & 6158 & 3000 & 730 & 1296 & 2860 & 4536 & 1668 & 86 \\
\hline Total & 2289 & 2084 & 6210 & 3062 & 779 & 1473 & 2913 & 4582 & 2226 & 728 \\
\hline \multicolumn{11}{|c|}{ CRUSTACEA } \\
\hline $\begin{array}{l}\text { Pachygrapsus gracilis (de Saussure, } \\
\text { 1858) }\end{array}$ & 3 & 0 & 4 & 0 & 1 & 2 & 5 & 11 & 1 & 0 \\
\hline $\begin{array}{l}\text { Pachygrapsus transversus (Gibbes, } \\
\text { 1850) }\end{array}$ & 0 & 0 & 0 & 0 & 0 & 0 & 0 & 1 & 0 & 0 \\
\hline Aratus pissoni (Warner 1967) & 0 & 0 & 0 & 0 & 0 & 0 & 1 & 0 & 0 & 0 \\
\hline Alpheus sp. & 0 & 0 & 0 & 1 & 0 & 0 & 0 & 0 & 0 & 0 \\
\hline Aoridae Morfo 1 & 0 & 0 & 0 & 0 & 0 & 0 & 6 & 0 & 0 & 0 \\
\hline Cf. Microdeutopus Morfo 1 & 0 & 0 & 0 & 0 & 1 & 0 & 0 & 0 & 0 & 0 \\
\hline Cf. Corophus Morfo 1 & 0 & 0 & 0 & 0 & 0 & 0 & 1 & 0 & 0 & 0 \\
\hline Cf. Erichtonius Morfo 1 & 0 & 0 & 0 & 0 & 0 & 0 & 1 & 0 & 0 & 0 \\
\hline Cf. Crangonyx spp. & 1 & 1 & 1 & 1 & 2 & 0 & 21 & 1 & 0 & 3 \\
\hline Gammaridae Morfo 1 & 12 & 0 & 34 & 7 & 14 & 24 & 23 & 8 & 26 & 27 \\
\hline Gammaridae Morfo 2 & 0 & 4 & 0 & 0 & 0 & 0 & 10 & 0 & 18 & 0 \\
\hline Gammaridae Morfo 3 & 0 & 1 & 0 & 0 & 0 & 0 & 2 & 0 & 2 & 1 \\
\hline Gammaridae Morfo 4 & 0 & 0 & 0 & 0 & 0 & 0 & 1 & 0 & 2 & 0 \\
\hline Gammaridae Morfo 5 & 0 & 2 & 0 & 0 & 0 & 3 & 1 & 2 & 8 & 4 \\
\hline Gammaridae Morfo 6 & 0 & 4 & 0 & 0 & 0 & 2 & 1 & 0 & 2 & 0 \\
\hline Gammaridae Morfo 7 & 0 & 0 & 0 & 0 & 0 & 0 & 2 & 1 & 2 & 0 \\
\hline Gammaridae Morfo 8 & 0 & 0 & 2 & 0 & 0 & 0 & 0 & 2 & 0 & 0 \\
\hline Gammaridae Morfo 9 & 0 & 0 & 0 & 0 & 0 & 0 & 0 & 0 & 1 & 0 \\
\hline Gammaridae Morfo 10 & 0 & 0 & 0 & 0 & 1 & 0 & 0 & 0 & 0 & 0 \\
\hline Hyale spp. & 65 & 67 & 1 & 5 & 0 & 6 & 48 & 26 & 17 & 0 \\
\hline Parhyale spp. & 43 & 50 & 10 & 2 & 0 & 1 & 1 & 24 & 3 & 0 \\
\hline Cf Listriella spp. & 0 & 2 & 0 & 0 & 0 & 0 & 43 & 0 & 0 & 5 \\
\hline Podoceropcis sp. & 0 & 0 & 0 & 0 & 0 & 0 & 0 & 0 & 1 & 0 \\
\hline Gonodactilidae Morfo 1 & 0 & 0 & 0 & 0 & 1 & 0 & 0 & 0 & 0 & 0 \\
\hline Balanus eburneus (Gould, 1841) & 0 & 0 & 0 & 0 & 0 & 0 & 2 & 16 & 2 & 5 \\
\hline Joeropsis spp. & 1 & 8 & 21 & 0 & 0 & 175 & 42 & 156 & 250 & 0 \\
\hline Postlarva Flabellifera & 0 & 0 & 0 & 0 & 0 & 3 & 0 & 0 & 0 & 23 \\
\hline Excorallana spp. & 6 & 2 & 0 & 40 & 24 & 13 & 2 & 4 & 26 & 3 \\
\hline Paracerceis spp. & 0 & 0 & 1 & 1 & 0 & 5 & 63 & 6 & 11 & 6 \\
\hline Gnatia sp. & 0 & 0 & 1 & 0 & 0 & 0 & 0 & 2 & 0 & 0 \\
\hline Ligia sp. & 1 & 7 & 0 & 0 & 0 & 0 & 0 & 1 & 0 & 0 \\
\hline Leptochelia sp. & 1 & 0 & 2 & 0 & 0 & 0 & 0 & 0 & 0 & 3 \\
\hline Leptochelidae Morfo 1 & 35 & 3 & 16 & 6 & 0 & 70 & 35 & 13 & 76 & 16 \\
\hline Leptochelidae Morfo 2 & 0 & 0 & 0 & 0 & 0 & 9 & 12 & 0 & 0 & 6 \\
\hline Leptochelidae Morfo 3 & 0 & 0 & 5 & 0 & 0 & 0 & 0 & 0 & 0 & 0 \\
\hline Pseudozeuxidae Morfo 1 & 153 & 12 & 188 & 50 & 0 & 205 & 150 & 99 & 253 & 56 \\
\hline Tanaidae Morfo 1 & 0 & 0 & 3 & 0 & 0 & 0 & 0 & 0 & 0 & 0 \\
\hline Total & 321 & 163 & 289 & 113 & 44 & 518 & 473 & 373 & 701 & 158 \\
\hline TOTAL & 2979 & 2335 & 6567 & 5574 & 1323 & 2469 & 3463 & 4991 & 3332 & 1142 \\
\hline
\end{tabular}


Tabla 2

Salinidad (ups) y temperatura $\left({ }^{\circ} \mathrm{C}\right)$ a los 103, 132, 161 y 188 días de muestreo en dos bahías en San Andrés Isla, 2003

Salinity (psu) and temperature $\left({ }^{\circ} \mathrm{C}\right)$ at 103, 132, 161 and 188 days of sampling in two bays at San Andres Island, 2003

\begin{tabular}{|c|c|c|c|c|c|c|c|}
\hline \multirow{3}{*}{ Días } & \multirow{3}{*}{ Parámetro } & \multicolumn{6}{|c|}{ Estaciones } \\
\hline & & \multicolumn{3}{|c|}{ Bahía Honda } & \multicolumn{3}{|c|}{ Bahía Hooker } \\
\hline & & E1 & $\mathrm{E} 2$ & E3 & E1 & $\mathrm{E} 2$ & E3 \\
\hline \multirow[t]{2}{*}{30} & UPS & 22,0 & 23,0 & 24,0 & 24,0 & 27,0 & 29,0 \\
\hline & ${ }^{\circ} \mathrm{C}$ & 29,7 & 29,0 & 29,9 & 29,6 & 30,0 & 29,7 \\
\hline 103 & UPS & 37,0 & 39,0 & 38,0 & 37,0 & 37,0 & 37,0 \\
\hline \multirow[t]{2}{*}{132} & UPS & 42,9 & 42,7 & 40,9 & 36,8 & 42,0 & 41,9 \\
\hline & ${ }^{\circ} \mathrm{C}$ & 29,0 & 31,8 & 31,3 & 31,2 & 31,9 & 31,3 \\
\hline \multirow[t]{2}{*}{161} & UPS & 36,0 & 37,0 & 38,0 & 32,3 & 36,0 & 33,0 \\
\hline & ${ }^{\circ} \mathrm{C}$ & 36,7 & 33,6 & 30,0 & 35,7 & 32,7 & 32,0 \\
\hline \multirow[t]{2}{*}{188} & UPS & 37,7 & 36,7 & & 35,8 & 32,3 & \\
\hline & ${ }^{\circ} \mathrm{C}$ & 33,9 & 33,8 & & 35,8 & 36,6 & \\
\hline
\end{tabular}

(equivalente al premuestreo) hasta el 132, con una ligera disminución hacia el 161. Hasta el final del estudio los valores se mantuvieron más o menos constantes. La temperatura aumentó desde el día 30 hasta el 161. A los 188 días no había habido mayor cambio (Tabla 2).

El análisis de similitud en el dendrograma reunió estaciones de diferentes lapsos. Se formaron dos grupos, I: K3(57), K3(161), O2(188), O3(161), O1(188); II: K2(57), K2(161), O2(132), O2(103), K3(103), O1(161), O2(161), O1(57), K3(132), O1(103), O1(132), O2(57), O3(132) (Fig. 3), que revelaron poca similitud entre la estación 1 de Bahía Hooker (K1) y las demás. El resultado del NMDS (Fig. 4) mostró que dicha estación estuvo más dispersa que las demás, principalmente en el esquema para anélidos con un nivel de estrés de representación más que razonable $(0,04)$, algunas veces acompañada por K2. Para el esquema de crustáceos en el mismo análisis esto no fue tan evidente, no se identificaron grupos de estaciones. Para moluscos, con un nivel de estrés de 0,01, solamente se dispersó KI (57), mostrando un proceso diferente. Teniendo en cuenta que los anélidos fueron los más abundantes, estas agrupaciones posiblemente se dieron por la aparición constante y frecuente de Spirorbis sp. especialmente, y por otras especies en menor cuantía, lo que no ocurrió en K1.

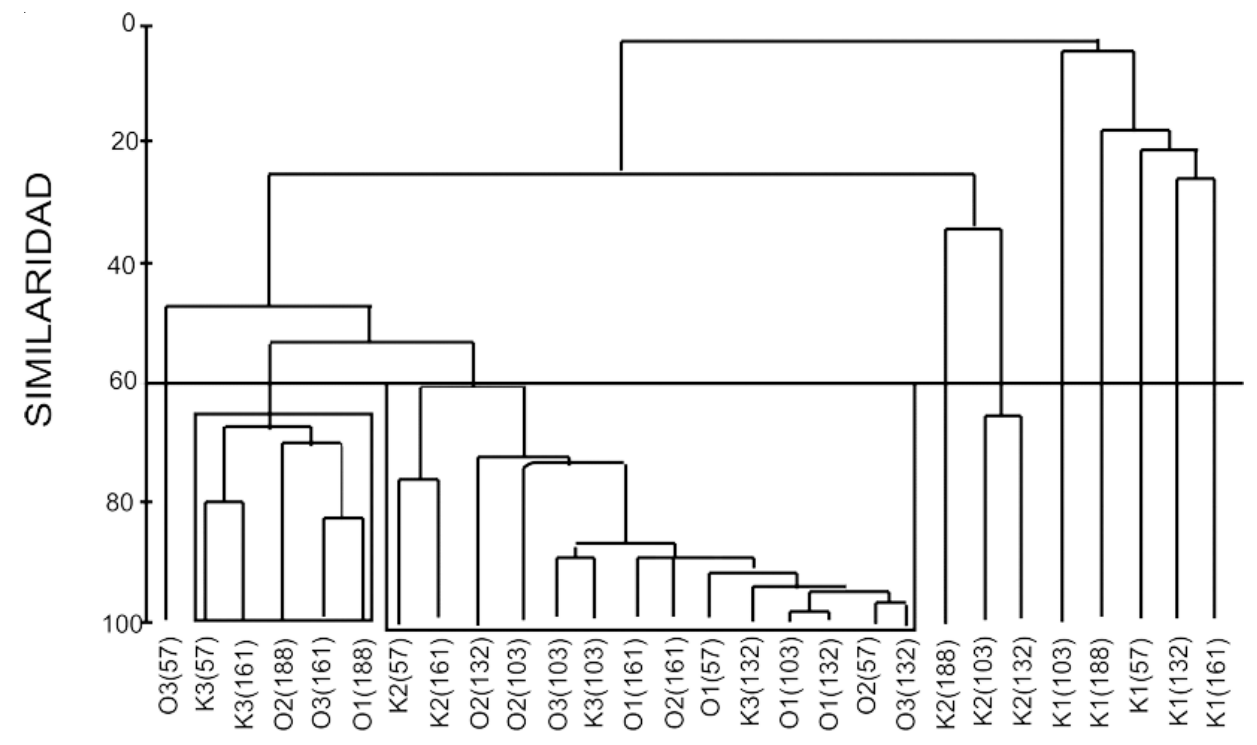

Figura 3

Dendrograma efectuado para las comunidades de crustáceos, poliquetos y moluscos desde febrero hasta mayo de 2003 en San Andrés Isla (Caribe colombiano), con la siguiente nomenclatura: O: Honda, K: Hooker, estación (1, 2 o 3), (días)

Dendrogram for community of crustaceans, polychaete worms, and molluscs from February through May 2003 in San Andres Island (Colombian Caribbean), with the following nomenclature: O: Honda, K: Hooker, station (1, 2 or 3), (days) 

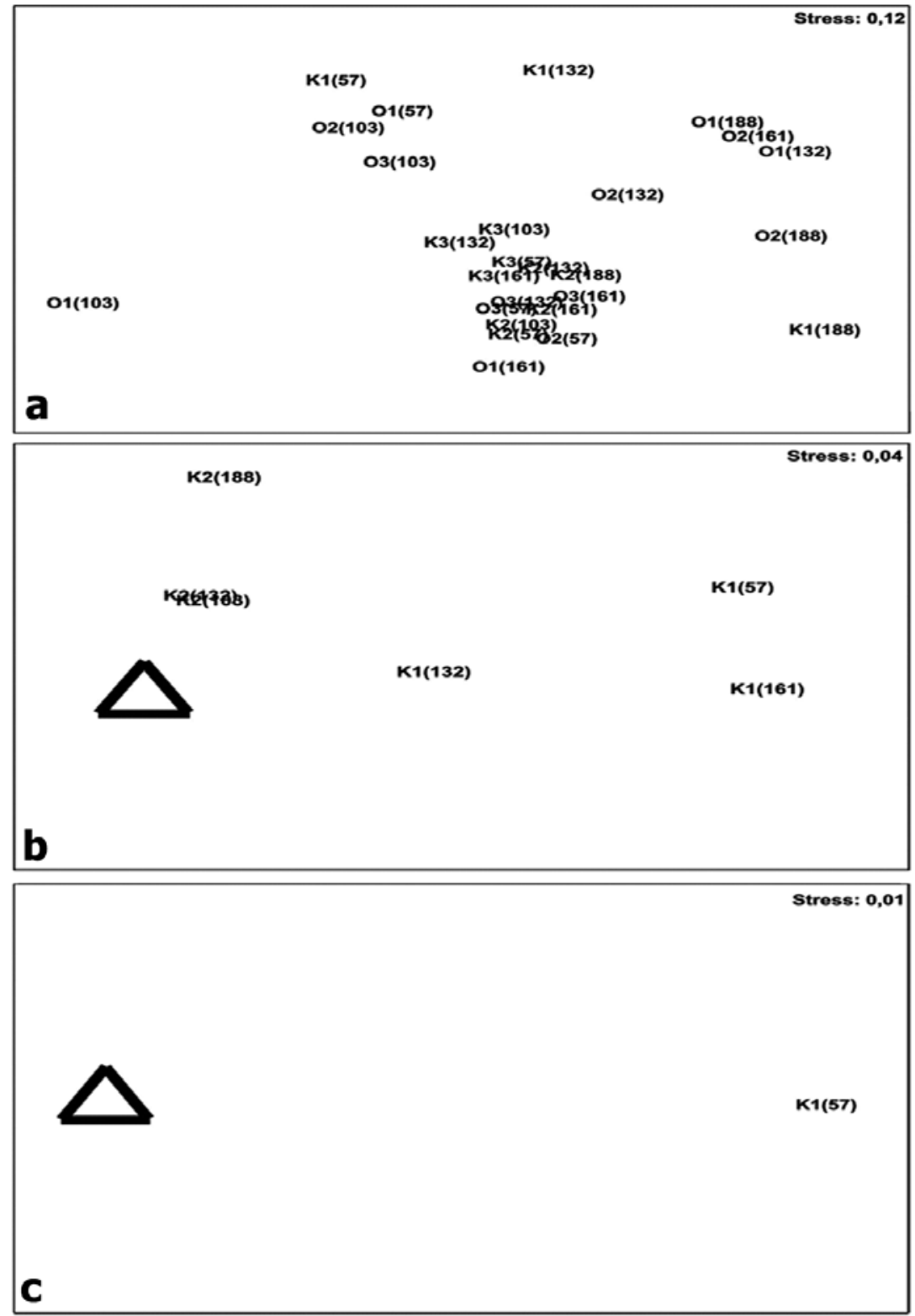

Figura 4

NMDS efectuado para los crustáceos (a), poliquetos (b) y moluscos (c) desde febrero hasta mayo de 2003 en San Andrés Isla (Caribe colombiano), con la siguiente nomenclatura: O: Honda, K: Hooker. La distribución de algunas estaciones en moluscos (O1(57), O2(57), O3(57), K2(57), K3(57), O1(103), O2(103), O3(103), K1(103), K3(103), O1(132), O2(132),

O3(132), K3(132), O1(161), O2(161), O3(161), K2(161), K3(161), O1(188), O2(188), K2(188)) y poliquetos (01(57), O2(57), O3(57), K2(57), K3(57), O1(103), O2(103), O3(103), K1(103), K2(103), K3(103), O1(132), O2(132), O3(132), K1(132),

K2(132), K3(132), O1(161), O2(161), O3(161), K1(161), K2(161), K3(161), O1(188), O2(188), K1(188)) se representó así:

\section{$\triangle$ porque todas estas estaciones estaban formando un solo grupo}

NMDS for crustaceans (a), polychaete worms (b), and molluscs (c) from February through May 2003 in San Andres Island (Colombian Caribbean), with the following nomenclature: O: Honda, K: Hooker. The distribution of some stations in mollusks (O1(57), O2(57), O3(57), K2(57), K3(57), O1(103), O2(103), O3(103), K1(103), K3(103), O1(132), O2(132), O3(132), K3(132), O1(161), O2(161), O3(161), K2(161), K3(161), O1(188), O2(188), K2(188)), and polychaete worms (O1(57), O2(57), O3(57), K2(57), K3(57), O1(103), O2(103), O3(103), K1(103), K2(103), K3(103), O1(132), O2(132), O3(132), K1(132), K2(132),

K3(132), O1(161), O2(161), O3(161), K1(161), K2(161), K3(161), O1(188), O2(188), K1(188)) were represented as: $\triangle$ because all of them were making the same group 
Tabla 3

Índices ecológicos aplicados a la abundancia de crustáceos, moluscos y anélidos de $R$. mangle en dos bahías de San Andrés Isla, 2003. Índices de riqueza de Margalef, uniformidad de Pielou, diversidad de Shannon-Wiener, predominio de Simpson, en cada bahía, estación y período muestreado

Ecological indexes applied to the abundance of crustaceans, mollusks and annelids over R. mangle in two bays of San Andres Island, 2003. Margalef's richness, Pielou's uniformity, Shannon-Wiener's diversity and Simpson's predominance indexes, in each bay station and sampled period

\begin{tabular}{|c|c|c|c|c|c|c|c|c|c|c|c|c|c|c|}
\hline \multicolumn{15}{|c|}{ Bahía Hooker } \\
\hline Período & \multicolumn{3}{|c|}{57} & \multicolumn{3}{|c|}{103} & \multicolumn{3}{|c|}{132} & \multicolumn{3}{|c|}{161} & \multicolumn{2}{|c|}{188} \\
\hline Índice Estación & E1 & E2 & E3 & E1 & E2 & E3 & E1 & E2 & E3 & E1 & E2 & E3 & E1 & E2 \\
\hline Riqueza & 2,817 & 1,990 & 2,140 & $* * * *$ & 2,671 & 4,068 & 3,693 & 3,778 & 2,825 & 2,954 & 3,062 & 3,048 & 2,078 & 3,144 \\
\hline Uniformidad & 0,952 & 0,574 & 0,470 & $* * * *$ & 0,716 & 0,232 & 0,961 & 0,780 & 0,129 & 0,964 & 0,438 & 0,512 & 0,685 & 0,491 \\
\hline Diversidad & 1,979 & 1,514 & 1,333 & 0,000 & 1,890 & 0,818 & 2,303 & 2,254 & 0,415 & 2,119 & 1,333 & 1,648 & 1,506 & 1,539 \\
\hline Predominio & 0,153 & 0,332 & 0,361 & 1,000 & 0,202 & 0,724 & 0,111 & 0,143 & 0,856 & 0,129 & 0,443 & 0,279 & 0,304 & 0,344 \\
\hline $\mathrm{N}^{\circ}$ Especies & 8 & 14 & 17 & 1 & 14 & 34 & 11 & 18 & 25 & 9 & 21 & 25 & 9 & 23 \\
\hline $\mathrm{N}^{\circ}$ Individuos & 12 & 688 & 1769 & 1 & 130 & 3332 & 15 & 90 & 4886 & 15 & 687 & 2630 & 47 & 1095 \\
\hline \multicolumn{15}{|c|}{ Bahía Honda } \\
\hline & \multicolumn{3}{|c|}{57} & \multicolumn{3}{|c|}{103} & \multicolumn{3}{|c|}{132} & \multicolumn{3}{|c|}{161} & \multicolumn{2}{|c|}{188} \\
\hline & E1 & E2 & E3 & E1 & E2 & E3 & E1 & E2 & E3 & E1 & E2 & E3 & E1 & E2 \\
\hline Riqueza & 1,482 & 0,914 & 2,724 & 0,592 & 1,308 & 2,597 & 1,069 & 3,073 & 3,134 & 0,421 & 2,231 & 2,284 & 1,018 & 2,726 \\
\hline Uniformidad & 0,155 & 0,118 & 0,491 & 0,027 & 0,466 & 0,229 & 0,053 & 0,444 & 0,090 & 0,265 & 0,221 & 0,306 & 0,359 & 0,572 \\
\hline Diversidad & 0,384 & 0,211 & 1,470 & 0,044 & 1,023 & 0,673 & 0,111 & 1,306 & 0,299 & 0,367 & 0,567 & 0,916 & 0,747 & 1,619 \\
\hline Predominio & 0,854 & 0,926 & 0,304 & 0,988 & 0,532 & 0,752 & 0,969 & 0,505 & 0,897 & 0,804 & 0,801 & 0,468 & 0,503 & 0,311 \\
\hline $\mathrm{N}^{\circ}$ Especies & 12 & 6 & 20 & 5 & 9 & 19 & 8 & 19 & 28 & 4 & 13 & 20 & 8 & 17 \\
\hline $\mathrm{N}^{\circ}$ Individuos & 1672 & 237 & 1070 & 858 & 453 & 1024 & 697 & 350 & 5520 & 1253 & 217 & 4104 & 969 & 354 \\
\hline
\end{tabular}

En cuanto a los índices ecológicos, en general los mayores valores de diversidad, riqueza y uniformidad analizados para los tiempos de muestreo estuvieron en Bahía Hooker. Por el contrario, para el predominio estuvieron en Bahía Honda. Los valores máximos y mínimos para diversidad y predominio en Hooker se dieron a los 132 días. En cambio en Bahía Honda los mayores y menores registros para diversidad, uniformidad y predominio estuvieron entre los días 57 y 103 días (Tabla 3).

\section{Discusión y conclusiones}

En general, los tres taxa estudiados se diferencian de otras comunidades descritas por Victoria \& Pérez (1979) y Reyes \& Campos (1992a, b) en la costa caribe colombiana debido a que muchas de las especies de la isla son particulares de esta zona (Londoño-Mesa et al. 2002, Moreno 2002, Vilardy \& Polanía 2002) posiblemente por encontrarse en una zona insular, a diferencia de los estudios nombrados que son a partir de zonas continentales, y son varias las especies no registradas en estas áreas, como Tricolia adamsi, T. bella, Assimenea succinea, Sabellastarte sp., Pilargis sp., entre otras.
Aunque desde una etapa temprana hay indicios de fauna asociada, a los 188 días se encontraron nuevas especies colonizadoras, lo que revela que la sucesión no termina en pocos meses y que, posiblemente, cambia con los ciclos climáticos de la isla. La disminución de las lluvias, con aumento en la salinidad desde el premuestreo y condiciones estables durante los días posteriores, dieron lugar a un aumento en la abundancia y diversidad (Victoria \& Pérez 1979).

Reyes \& Campos (1992a, b) observaron que en la Ciénaga Grande de Santa Marta y parte del Parque Tayrona hubo un incremento en la abundancia de las especies asociadas a partir de los periodos de baja salinidad y lluvias constantes. Sin embargo, también existen especies tolerantes a los cambios de salinidad como por ejemplo Isognomon alatus, Neanthes succinea, Pachygrapsus gracilis, entre otras, según lo registrado por Victoria \& Pérez (1979), de hecho, estas especies fueron observadas a lo largo de la sucesión y, posiblemente se establecen principalmente por la disponibilidad de sustrato y la oferta alimenticia.

En cuanto al patrón de distribución de las especies en el proceso de sucesión, la fauna asociada a las raíces de 
mangle rojo se dispersa en forma de parche, de una manera totalmente azarosa e irregular en toda el área de estudio y desde los comienzos del proceso, con diferencias de raíz a raíz y entre sectores de manera amplia, al igual que en otros estudios (Sutherland 1980, Inclán 1989, Reyes \& Campos 1992a, b, Farnsworth \& Ellison 1996). Sin embargo, las estaciones 1, 2 y 3 de Bahía Honda y 3 de Bahía Hooker guardan a lo largo del estudio similitud entre especies. La sucesión en las dos bahías de San Andrés Isla indica que el proceso es diferente. Las diferencias, de espacio principalmente, se dan desde el interior de Bahía Honda hacia el exterior de Bahía Hooker, y la parte media e interna de Hooker como otra zona. Cada parte de las bahías muestra características específicas en cuanto al sustrato y la profundidad que producen la separación de las dos zonas mencionadas.

Bahía Hooker, cuerpo de agua semicerrado por modificaciones efectuadas en la entrada de la bahía para la construcción de una planta de energía y el muelle, fue sometida a contaminación térmica y de hidrocarburos, y aún presenta concentraciones de aromáticos totales (Vilardy \& Polanía 2002). Sufre contaminación de aguas residuales por los asentamientos a sus alrededores (Romero-Murillo 2004). Bahía Honda es un cuerpo abierto que forma una pequeña laguna costera y se encuentra bordeada en su costado norte por sedimentos carbonatados, con menor profundidad en la boca e influencia permanente de corrientes mareales; afectada parcialmente por aguas servidas (CORALINA 1997).

Para anélidos es notoria la separación que hay entre la estación 1 de Hooker y las demás, que puede ser resultado de las condiciones ambientales, corrientes, vientos y precipitación; K1 presenta una situación característica de pocas especies con poca abundancia, sólo las especies adaptadas a condiciones extremas y difíciles se desarrollan allí, como presencia de desechos de hidrocarburos, aguas servidas y disminución del nivel de agua, o típicas de la zona de mangle. Éstas modulan la comunidad para que sea diferente a las demás zonas y su colonización se produzca de otra manera. Mientras, para los crustáceos esto no es tan visible y las agrupaciones pueden responder a la dispersión larval (Farnsworth \& Ellison 1996, Lalana \& Pérez 1985). Para moluscos el proceso se desarrolló de manera similar en varias estaciones en diferentes períodos muestreados, a excepción de K1 a los 57 días.

Los anélidos son dominantes y muestran un patrón de sucesión, caracterizado por la ocupación del espacio; la competencia lleva a la dominancia en este caso, desplazando comunidades como la de los moluscos. En este trabajo, Spirorbis sp. y otros fueron colonizadores pioneros y los tubos abandonados se convirtieron en sustrato nuevo (Inclán 1989, Bruno et al. 2003), hacia el día 103. Desde el interior de Bahía Honda hasta la parte externa de Bahía Hooker cierta competencia favoreció a esta especie, pues rápidamente ocupó el espacio disponible e impidió que otras, como moluscos necesitados de sustrato estable, permanecieran (Reyes \& Campos 1992a). Esto indicaría un período inicial de sucesión, en el que especies de estrategia R (Spirorbis sp.) son más numerosos que los de estrategia K (MetcalfeSmith et al. 1998).

Por otra parte, K1 presenta una situación característica de pocas especies con poca abundancia. Sólo las especies adaptadas a condiciones extremas y difíciles se desarrollan allí, como presencia de desechos de hidrocarburos, aguas servidas y disminución del nivel de agua, o típicas de la zona de mangle. Londoño-Mesa et al. (2002) ya lo habían mencionado para los anélidos como Neanthes sp., y Vilardy \& Polanía (2002) para moluscos, como Neritina virginea.

Se observó que los crustáceos son transitorios, se asientan donde hay un sustrato estable, diferentes fuentes alimenticias y condiciones ambientales propicias para mantenerse, al menos durante alguna etapa de su ciclo de vida. La presencia de ciertos crustáceos perforadores evidencia el sustento que representan las raíces como sustrato para establecerse y como alimento (de manera directa porque se alimentan de ellas o del sedimento que se adhiere a ellas, y de manera indirecta porque se alimentan de organismos que llegan a las raíces).

Las algas en las estaciones 3 de ambas bahías disminuye la competencia de forma indirecta por espacio y aumenta la cantidad de nichos, lo que hace que un mayor número de especies se encuentren en las estaciones para las tres comunidades (Jones et al. 1997). Así también la cercanía con otros ecosistemas (arrecifes de coral y praderas de pastos) permite el tránsito de diversas especies (Cruz-Ábrego et al. 1994), como Excorallana spp. y Joeropsis spp., que es lo que presentan estas estaciones.

El comportamiento general de los tres grupos estudiados mostró que la diversidad y la uniformidad fueron mayores en Bahía Hooker. La taxocenosis Mollusca, Annelida y Crustacea se encuentra en una etapa inicial de sucesión, alterada por el cambio en la salinidad (a los 132 días), que incrementó la abundancia de estos organismos en las dos bahías. Spirorbis sp. y Petaloconchus sp. fueron las especies más abundantes, pero disminuyen después de los 132 días. 
Un aporte importante de sedimentos calcáreos favorece el establecimiento de ciertos moluscos, generalmente de poca movilidad, desde la parte interna de Bahía Honda hasta la externa de Bahía Hooker. Entre éstos es posible citar Ciclostremiscus sp., Petaloconchus sp., Modulus modulus, Caecum cornucopidae (un gasterópodo que habita usualmente en el sedimento) y Chione cancellata. Muchos otros son característicos de los fondos lodosos, como Melampus coffeus (un gasterópodo pulmonado) y Prunum sp., y otros, como Littorina angulifera, Isognomon alatus (adherida al sustrato por un biso) y Mytilopsis salei, son propios de las raíces de mangle y fueron encontrados en K1.

El proceso de sucesión puede estar influenciado por la geomorfología de las bahías. Especies filtradoras, como por ejemplo Spirorbis sp., pueden establecerse fácilmente y la obtención de alimento es más sencilla en aquellas áreas en donde el flujo de las corrientes es mayor, posiblemente por la variación en los vientos, en el caso de bahía Honda. Su disposición como cuerpo abierto permite tener un mayor flujo de corrientes y los vientos llevan al movimiento de partículas, mientras que en bahía Hooker el cuerpo de agua semicerrado lleva a condiciones más difíciles y a un estancamiento de las aguas que fluctúan poco a través del canal de entrada (Garay \& Castro 1990).

Si bien no hay datos que lo sustenten, otros factores ambientales (salinidad y temperatura) y antrópicos (presencia de lodos aceitosos y aguas servidas de la antigua planta eléctrica y los actuales barrios aledaños, los valores encontrados por el programa CARIPOL/Colombia hasta 1990 arrojaron un resultado de $15.88 \mu \mathrm{l}^{-1}$ para las aguas superficiales en la zona de San Luis sensu Garay \& Castro 1990, García-Hansen et al. 2002) también podrían influir en el desarrollo de la taxocenosis (Hensel et al. 2002). Los cambios de las temporadas de sequía y lluvias, que varían las concentraciones de salinidad y, posiblemente, también las de partículas disueltas, producen un cambio en la composición de especies. En contraste, Reyes \& Campos (1992a) consideran que la agrupación de diferentes épocas puede deberse a una poca relación con la fluctuación de los actores ambientales.

En cuanto a trabajos efectuados en la misma zona, Londoño-Mesa et al. (2002) en dos períodos estacionales diferentes (época seca y de lluvias en 1998) encontraron 49 especies. Moreno (2002), quien trabajó en un período similar, halló solamente 54 especies. Polydora sp., que fue registrada por Moreno (2002) en una temporada lluviosa, no ha sido registrada en otros trabajos en el área ni en éste. Reyes \& Campos (1992a) y Hernández-
Alcántara \& Solís-Weiss (1995) han señalado que la precipitación puede ser importante en la regulación de esta taxocenosis y, en este caso, explicaría la aparición de Polydora sp. sólo en una temporada lluviosa. En temporada seca prosperan otras poblaciones, como Sabellastarte spp., que se mantienen hasta que una nueva temporada de lluvias disminuye la salinidad, entre 5 a 20 ppm (ver Londoño-Mesa et al. 2002, Moreno 2002, Vilardy \& Polanía 2002).

Las tasas variables de reclutamiento de los representantes de los taxa considerados, seguramente determinan en algún grado las diferencias halladas entre temporadas (Farnsworth \& Ellison 1996), y podrán estar relacionadas con las corrientes y aumento en la precipitación, así como con los cambios mareales (Farnsworth \& Ellison 1996, Wethey 20044).

En esta etapa inicial del proceso de sucesión las comunidades no guardan homogeneidad, el cambio en períodos sucesivos no es muy evidente, pero en cuanto hay una variación climática (en precipitación, salinidad, temperatura) se desencadena una serie de nuevos procesos en la colonización de las raíces, con nuevas poblaciones y números indeterminados de individuos.

A partir de este trabajo y para siguientes investigaciones en la zona y en áreas similares, es necesario tener en cuenta que las comunidades acá estudiadas en un proceso sucesional se ven afectadas por factores antrópicos y ambientales, que determinarán el tiempo de recuperación en ambientes que han sufrido algún tipo de disturbio.

\section{Agradecimientos}

Los autores agradecen a las universidades Nacional de Colombia sede Caribe por sus instalaciones, equipos y todo su apoyo para la realización de este trabajo, a la de Bogotá Jorge Tadeo Lozano y a la Militar Nueva Granada por los equipos y el laboratorio. También agradecen la colaboración de los biólogos Juan Laverde en la identificación de anélidos, Ángela López y Néstor Campos en anfípodos y, en general, crustáceos, y la Dra. Mónica Puyana en moluscos. De igual manera, a los evaluadores y editores de la revista por su dedicación y aportes a este trabajo.

\footnotetext{
${ }^{4}$ Wethey D. 2004. Wave forces in the surf zone: Implications for larval settlement. The $6^{\text {th }}$ International Larval Biology Conference, p. 26. Hong Kong, China.
} 


\section{Literatura citada}

Álvarez-León R \& J Polanía. 1996. Los manglares del Caribe colombiano: Síntesis de su conocimiento. Revista de la Academia Colombiana de Ciencias Exactas, Físicas y Naturales 20(78): 447-464.

Batista VE. 1980. Estudio de las comunidades que habitan las raíces del mangle rojo Rhizophora mangle L. de Punta Galeta, costa atlántica de Panamá. Trabajo de pregrado. Universidad Jorge Tadeo Lozano. Bogotá, 124 pp.

Bousfield E. 1973. Shallow-water Gammaridean amphipoda of New England, 313 pp. Cornell University Press. National Museums of Canada, Ontario.

Bruno JF, JJ Stachowicz \& MD Bertness. 2003. Inclusión of facilitation into ecological theory. Trends in Ecology \& Evolution 18: 119-125.

Buitrago MF \& LF Cuartas. 2003. Caracterización y zonación de las comunidades de manglar en San Andrés Isla, Caribe colombiano. Trabajo de pregrado. Universidad Nacional de Colombia sede Medellín, Medellín, 61 pp.

CORALINA. 1997. Boletín de la Red de Monitoreo de las Zonas Costeras. San Andrés Colombia N² 2-6: 8-12.

CORALINA. 2003. Plan de manejo del sistema regional de áreas marinas protegidas-Parte I. V Componente Biótico, 63-210 pp. San Andrés Isla, Colombia.

Cruz-Ábrego FM, P Hernández-Alcántara \& V Solís-Weiss. 1994. Estudio de la fauna de poliquetos (Annelida) moluscos (Gastropoda y Bivalvia) asociada con ambientes de pastos marinos Thalassia testudinum y manglares (Rhizophora mangle) en la Laguna de Términos, Campeche, México Anales del Instituto de Ciencias del Mar y Limnología de la Universidad Nacional Autónoma de México 21: 1-13.

De León-González J. 2001. Primer taller de taxonomía y evolución de poliquetos (Nereididae) 33 pp. ECOSUR, Quintana Roo.

Díaz JM \& M Puyana. 1994. Moluscos del Caribe colombiano. Un catálogo ilustrado, 291 pp. COLCIENCIAS / Fundación Natura / INVEMAR, Santa Marta.

Díaz JM, J Garzón-Ferreira \& S Zea. 1995. Los arrecifes coralinos de la San Andrés Isla, Colombia: Estado actual y perspectivas para su desarrollo. 150 pp. Academia Colombiana de Ciencias Exactas. Físicas y Naturales, Colecciones. Jorge Álvarez Lleras 7, Santafé de Bogotá.

Díaz JM, G Díaz-Pulido, J Garzon-Ferreira, J Geister, JA Sánchez \& S Zea. 1996. Atlas de los arrecifes coralinos del Caribe colombiano. I. Complejos Arrecifales Oceánicos. 2: 1-83 INVEMAR Serie de Publicación Especial.

Farnsworth E \& A Ellison. 1996. Scale-dependent spatial and temporal variability in biogeography of mangrove root epibiont communities. Ecological Monographs 66(1): 4566.

Fauchald K. 1975. Clave de poliquetos panameños con la inclusión de una clave para todas las familias del mundo. Boletín Oceanográfico Universidad de Oriente 14(1): 7194.

Fauchald K. 1977a. The Polychaete Worms: Definitions and Keys to the Orders, Families and Genera. Natural History Museum of Los Angeles County. Science Series 28: 1-188.

Fauchald K. 1977b. Polychaetes from intertidial areas in Panama, with a review of precious shallow. water records. Smithsonian Contributions to Zoology 221: 1-81.

Garay J \& LA Castro. 1990. Avances del programa Caripol/ Colombia 1985-1990. Memorias del VII Seminario Nacional de Ciencias y Tecnologías del Mar (Cali), pp. 225-230. Comisión Colombiana de Oceanografía, Bogotá.

Garay J, D Castillo, J Aguilera, L Niño, M de La Pava, W López \& G Márquez. 1988. Estudio oceanográfico del área insular y oceánica del Caribe colombiano, Archipiélago de San Andrés y Providencia y Cayos Vecinos. Boletín Científico Centro de Investigaciones Oceanográficas e Hidrográficas 9: 3-73.

García-Barriga, EJ Hernández, I Jaramillo, LE Mora, P Pinto \& PM Ruiz. 1985. La Isla de San Andrés: Contribución al conocimiento de su ecología, flora, fauna y pesca, 152 pp. Instituto de Ciencias Naturales. Facultad de Ciencias de la Universidad Nacional de Colombia. Dirección de Divulgación Cultural, Bogotá.

García-Hansen I, J Gaviria-Chiquazuque, MC PradaTriana \& R Álvarez-León. 2002. Producción de hojarasca de los manglares de la Isla de San Andrés, Caribe colombiano. Revista de Biología Tropical 50(1): 273-291.

González NE. 1998. Taxonomía de moluscos. Curso dictado en el proyecto ECOSUR Bentos costeros del Caribe Mexicano: Línea de base para estudios a largo plazo, 39 pp. CONACYT, Chetumal.

Gutu M \& J Sieg. 1999. Ordre des tanaïdacés (Tanaidacea Hansen, 1895). En: Forest J (ed), Traité de Zoologie. Anatomie, Systématique, Biologie. Tome 7, Crustacés, Fascicule 3A, Péracarides. Memoires de l'Institut Oceanographique Monaco 19: 353-389.

Harper DE. 1971. Key to the Polychaetus annelids of the northwestern Gulf of México, 90 pp. Moody College of Marine Science, Galveston.

Hensel P, E Proffitt, P Delgado, G Shigenaka, R Yender, R Hoff \& A Mearns. 2002. Oil spills in mangroves, pp. 5668. National Oceanic and Atmospheric Administration (NOAA) Ocean Service - Office of Response and Restoration, Washington. 
Hernández-Alcántara P \& V Solis-Weiss. 1995. Algunas comunidades macrobénticas asociadas al manglar en laguna de Términos, Golfo de México. Revista de Biologia Tropical 43: 117-129.

Inclán RR. 1989. Ecología de la epibiosis en las raíces inmersas de Rhizophora mangle en Bahía de la Ascensión, Quintana Roo, México. Ciencias Marinas 15(1): 1-20.

Jones C, J Lawton \& M Shachak. 1997. Positive and negative effects of organisms as physical ecosystems engineers. Ecology 78(7): 1946-1957.

Lacerda LD, JE Conde, B Kjerfve, R Álvarez-León, C Alarcón \& J Polanía. 2001. American Mangroves. de Lacerda LD (ed), Mangrove Ecosystem. Function and Management. pp. 1-62. Springer-Verlag, Berlín.

Lalana R \& M Pérez. 1985. Estudio cualitativo y cuantitativo de la fauna asociada a Rhizophora mangle en la Cayera. Revista de Investigaciones Marinas de la Habana 6(2-3): 45-58.

Leyton LM \& FE Delgado. 1992. Fauna asociada a las raíces de mangle rojo. Plan de monitoreo Golfo de Morrosquillo $3^{\mathrm{a}}$ fase. Informe Final, 47 pp. Ecopetrol, Bogotá.

Londoño-Mesa M, J Polanía \& I Vélez. 2002. Polychaetes of the mangrove- fouling community at the Colombian Archipiélago of San Andrés and Old Providence, Western Caribbean. Wetlands Ecology and Management 10: 227232.

Metcalfe-Smith JL, SK Staton, GL Mackie \& NM Lane. 1998. Changes in the biodiversity of freshwater mussels in the Canadian waters of the lower Great Lakes drainage basin over the past 140 years. Journal of Great Lakes Research 24(4): 845-858.

Moreno P. 2002. Taxocenosis Anellida-Mollusca-Crustacea asociada a las raíces sumergidas de Rhizophora mangle $\mathrm{L}$. en San Andrés Isla, Caribe colombiano. Trabajo de pregrado Universidad de Bogotá Jorge Tadeo Lozano, Bogotá, 57 pp.

Nonato E \& J Luna. 1970. Anélidos poliquetas do Nordeste do Brasil, I, Poliquetas bentónicos da costa de allagunas e Sergipe. Boletim Instituto Oceanográfico São Paulo 19: $1-144$.

Odum E. 1972. Ecología, 639 pp. Ed. Omega, Barcelona.

Ortiz M. 1994. Clave gráfica para la identificación de familias y géneros de anfípodos del suborden Gammaridea del Atlántico occidental tropical. Anales del Instituto de
Investigaciones Marinas y Costeras Punta Betín 23: 59101.

Reyes R \& N Campos. 1992a. Moluscos, anélidos y crustáceos asociados a las raíces de Rhizophora mangle Linnaeus, en la región de Santa Marta, Caribe colombiano. Caldasia 17(1): 133-148.

Reyes R \& N Campos. 1992b. Macroinvertebrados colonizadores de raíces de Rhizophora mangle en la Bahía de Chengue, Caribe colombiano. Anales del Instituto de Investigaciones Marinas y Costeras Punta Betín 21: 101116.

Rodríguez G. 1980. Los Crustáceos Decápodos de Venezuela, pp. 377-381. Centro de Ecología, Instituto Venezolano de Investigaciones Científicas, Caracas.

Romero-Murillo P. 2004. Composición taxonómica y proceso sucesional de la fauna asociada a las raíces sumergidas de Rhizophora mangle (mangle rojo) de San Andrés isla. Trabajo de pregrado, Universidad de Bogotá Jorge Tadeo Lozano, Bogotá, 98 pp.

Rouse G \& F Pleijel. 2001. Polychaetes, 354 pp. Oxford University Press, Oxford.

Salazar-Vallejo SI \& LF Carrera-Parra. 1997. Eunicidos (Polychaeta) del Caribe Mexicano con claves para las especies del Gran Caribe: Fauchaldius, Lysidice, Marphysa, Nematonereis y Palola. Revista Biología Tropical 45(4): 1481-1498.

Salazar-Vallejo SI \& LF Carrera-Parra. 1997. Taxonomía de poliquetos (Annelida, Polychaeta), 64 pp. CONACYTECOSUR, Chetumal.

Salazar-Vallejo S, J de León-González \& H SalaicesPolanco. 1988. Poliquetos (Annelida: Polychaeta), 212 pp. Universidad de Baja California Sur. Libros Universitarios, Baja California.

Sutherland JP. 1980. Dynamics of the epibenthic community of roots of the mangrove Rhizophora mangle, at Bahía de Buche, Venezuela. Marine Biology 58: 75-84.

Victoria C \& M Pérez. 1979. Los taxa Annelida, Mollusca y Crustacea en las raíces sumergidas del mangle rojo de dos áreas costeras del Caribe colombiano. Informe del Museo del Mar, Bogotá. 21: 1-23

Vilardy S \& J Polanía. 2002. Mollusc fauna of the mangroveroot fouling community at the Colombian Archipelago of San Andrés and Old Providence. Wetlands Ecology and Management 10: 273-282. 